\title{
Circular RNA Circ100084 functions as sponge of miR-23a-5p to regulate IGF2 expression in hepatocellular carcinoma
}

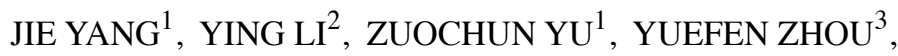 \\ JIANFEI TU ${ }^{4}$, JIAN LOU ${ }^{3}$ and YONGHUI WANG ${ }^{3}$ \\ Departments of ${ }^{1}$ Infectious Disease, ${ }^{2}$ Stomatology, ${ }^{3}$ Oncology and ${ }^{4}$ Intervention, \\ Lishui Municipal Central Hospital, Lishui, Zhejiang 323000, P.R. China
}

Received January 18, 2019; Accepted March 13, 2020

DOI: $10.3892 / \mathrm{mmr} .2020 .11069$

\begin{abstract}
Hepatocellular carcinoma (HCC) has become a major cause of cancer-related mortality worldwide. Circular RNAs (circRNAs) are non-coding RNAs that serve important roles in multiple cancers. However, the role of circRNAs in HCC remains largely unknown. In the present study, a circRNA microarray dataset of HCC samples, GSE97332, was downloaded from the gene expression omnibus database. Following data preprocessing, differentially expressed circRNAs between HCC tissues and normal tissues were determined using GEO2R. The circRNA-miRNA interactions were predicted by the miRanda database. The miRTarbase database was used to search for target genes of the miRNAs. A circRNA-miRNA-mRNA network was constructed using Cytoscape based on the obtained circRNA, miRNA and mRNA. In this network, the upregulated circRNA hsa_circRNA_100084 was found to be involved in a competing endogenous relationship of hsa circRNA_100084-hsa-miR-23a-5p-insulin-like growth factor 2 (IGF2). The differential expression of hsa_circRNA_100084, hsa-miR-23a-5p and IGF2 in HCC tissues and liver cancer cells was validated by reverse transcription-quantitative PCR. Additionally, the interactions between hsa-miR-23a-5p with hsa_circRNA_100084 and IGF2 were validated by dual-luciferase reporter assays. Knocking down hsa_circRNA_100084 inhibited the proliferation, migration and invasion of liver cancer cells, while the simultaneous overexpression of $I G F 2$ reversed the effects of hsa_circRNA_100084 knockdown. The results show that hsa_circRNA_100084 could promote the expression of IGF2 by acting as a sponge of hsa-miR-23a-5p in liver cancer cells.
\end{abstract}

\section{Introduction}

Hepatocellular carcinoma (HCC) is the most common type of liver cancer and is the leading cause of cancer-related deaths

Correspondence to: Dr Yonghui Wang, Department of Oncology, Lishui Municipal Central Hospital, 289 Kuocang Road, Lishui, Zhejiang 323000, P.R. China

E-mail: 1szxyywyh123@163.com

Key words: hepatocellular carcinoma, circular RNA, miRNA, sponge worldwide $(1,2)$. Although surgical resection combined with post-surgery radio-chemotherapy has achieved great progress, the median survival time for patients with HCC remains unsatisfactory (3). It is estimated that $\sim 700,000$ individuals succumb to HCC each year globally (4). Therefore, further investigations into the molecular basis of $\mathrm{HCC}$ are required to explore innovative targets for its diagnosis and treatment.

Circular RNA (circRNA) is a newly discovered class of endogenous noncoding RNAs that are characterized by a covalently closed continuous loop (5). Due to their special structure, circRNAs are highly evolutionarily conserved and stable (6). Recent studies have indicated that circRNAs may serve important roles in driving cancer initiation and progression, and have the potential to serve as biomarkers for predicting cancer progression $(7,8)$. Accumulating evidence has demonstrated that circRNAs regulate HCC progression and serve as potential biomarkers for predicting cancer prognosis (9-15). However, a considerable number of circRNAs remain to be elucidated in $\mathrm{HCC}$.

Insulin-like growth factor 2 (IGF2) is a genomic imprinting gene involved in development and growth, which is located on the short arm of chromosome 11 (16). This gene is a paternally imprinted growth factor regulated by four promoters. During fetal stages, the expression of $I G F 2$ is monoallelically regulated from 3 promoters ( $\mathrm{P} 2, \mathrm{P} 3$ and $\mathrm{P} 4)$ in human liver and in adults, its expression is regulated by both alleles of promoter P1 (17). Although IGF2 is highly active during fetal development, it is much less active after birth (18). However, studies have suggested that IGF2 overexpression occurs in numerous types of cancers and is associated with resistance to chemotherapy and a worse prognosis $(18,19)$. This might be partly explained by the reactivation of $I G F 2$ transcription from the fetal-specific promoters or demethylation of its fetal promoter $(20,21)$. However, further studies are still needed to elucidate the mechanisms of $I G F 2$ overexpression in HCC.

Previous studies have demonstrated that circRNAs may function as competing endogenous RNAs (ceRNA) by sponging miRNAs to regulate target gene expression (22-24). In a previous study, Han et al (25) characterized the expression profile of circRNAs in human HCC tissues and paired adjacent liver tissues. They demonstrated that circMTO1 suppresses HCC progression by acting as a sponge for oncogenic miR-9 to promote $\mathrm{p} 21$ expression. In the present study, the sequencing 
data used in the study of Han et al was downloaded from the Gene Expression Omnibus (GEO) database and these data were re-analyzed using a series of bioinformatics methods. A ceRNA network was constructed and IGF2 was found to be involved in a ceRNA network of hsa_circRNA_100084-h sa-miR-23a-5p-IGF2. The present study further validated the ceRNA association in HCC tissues and liver cancer cells.

\section{Materials and methods}

RNA sequencing data. The expression profile of lncRNAs in human HCC was downloaded from the GEO database (26) (accession number: GSE97332), which was deposited by Han et al (25). This dataset contained seven pairs of HCC tumor tissues and matched non-tumor tissues and was based on the Agilent-069978 Arraystar Human CircRNA microarray V1 platform. Original expression data as well as the platform probes annotation files were downloaded.

Identification of differentially expressed (DE)-circRNAs in $H C C$. The original expression profiles of HCC and normal tissues were analyzed using GEO2R (https://www.ncbi.nlm. nih.gov/geo/geo2r/), which is an online tool for processing data using GEO queries and limma packages (27) in $\mathrm{R}$ from the Bioconductor project (28). The raw data were preprocessed by background correction and normalization by $\log 2$ transformation. The raw P-value was adjusted by the Benjamini and Hochberg method to a false discovery rate (FDR). The circRNAs with thresholds of FDR $<0.05$ and |logfold change (FC) $\mid>2$ were considered as DE-circRNAs. Heatmap for the top 10 upregulated circRNAs and the top 10 downregulated circRNAs were constructed using pheatmap method in R package (http://finzi.psych.upenn. edu/R/library/pheatmap/html/pheatmap.html).

Construction of a circRNA-miRNA-mRNA network. The top 10 upregulated circRNAs and top 10 downregulated circRNAs were selected for further analysis. Since circRNA nomenclature differs among platforms, the present study first mapped the probe sequences of the top 10 upregulated circRNAs and top 10 downregulated circRNAs into circBase (http://www.circbase.org/cgi-bin/webBlat). The associations with the highest matching score were selected. The miRNAs related to the $20 \mathrm{DE}$-circRNAs were predicted by miRanda v.3.3a (http://www.microrna.org/microrna/home.do) with the criteria of Score $\geq 140$ and Energy $\leq-10$ (29).

The HCC-associated miRNAs were searched on the miR2Disease (www.mir2disease.org) database using 'hepatocellular carcinoma' as key words. Then, the overlapping miRNAs of HCC-associated miRNAs and the predicted miRNAs in the previous step were noted for further study.

Prediction of miRNA target genes. The online tool miRWalk2.0 (http://zmf.umm.uni-heidelberg.de/apps/zmf/mirwalk2/) (30) was used for predicting the target genes of miRNAs in 7 databases: miRWalk (http://mirwalk.umm.uni-heidelberg. de/), miRanda (http://www.microrna.org/microrna/home. do), miRDB (http://mirdb.org/), miRMap (https://mirmap. ezlab.org/), miRNAMap (http://mirnamap.mbc.nctu.edu.tw/), RNA22 (https://cm.jefferson.edu/rna22/) and TargetScan
Table I. Clinicopathological characteristics of patients with HCC cancer in this study $(n=37)$.

\begin{tabular}{|c|c|}
\hline Characteristic & Patients, n $(\%$ \\
\hline \multicolumn{2}{|l|}{ Age, years } \\
\hline$<60$ & $16(43.2 \%)$ \\
\hline$\geq 60$ & $21(56.8 \%)$ \\
\hline \multicolumn{2}{|l|}{ Sex } \\
\hline Male & $23(62.2 \%)$ \\
\hline Female & $14(37.8 \%)$ \\
\hline \multicolumn{2}{|c|}{ Serum AFP, ng/ml } \\
\hline Negative & $13(35.1 \%)$ \\
\hline Positive & $24(64.9 \%)$ \\
\hline \multicolumn{2}{|l|}{ Smoking } \\
\hline Negative & $18(48.6 \%)$ \\
\hline Positive & $19(51.4 \%)$ \\
\hline \multicolumn{2}{|l|}{ Alcohol } \\
\hline Negative & $27(73 \%)$ \\
\hline Positive & $10(27 \%)$ \\
\hline \multicolumn{2}{|l|}{ Cirrhosis } \\
\hline Present & $28(75.7 \%)$ \\
\hline Absent & $9(24.3 \%)$ \\
\hline \multicolumn{2}{|l|}{ T stage } \\
\hline $\mathrm{T} 1-\mathrm{T} 2$ & $15(40.5 \%)$ \\
\hline T3-T4 & $22(59.5 \%)$ \\
\hline \multicolumn{2}{|c|}{ Regional lymph node metastasis } \\
\hline Yes & $16(43.2 \%)$ \\
\hline No & $21(56.8 \%)$ \\
\hline \multicolumn{2}{|c|}{ Distant metastasis } \\
\hline Yes & $11(29.7 \%)$ \\
\hline No & $26(70.3 \%)$ \\
\hline \multicolumn{2}{|l|}{ Tumor size } \\
\hline$<5 \mathrm{~cm}$ & $26(70.3 \%)$ \\
\hline$\geq 5 \mathrm{~cm}$ & $11(29.7 \%)$ \\
\hline
\end{tabular}

AFP, $\alpha$ fetal protein; HCC, hepatocellular carcinoma.

(http://www.targetscan.org/vert_72/). The target genes predicted by at least 6 databases were retained. To further filter the target genes, miRanda was used to calculate the score and Energy for the combination of miRNA-mRNA (3'-untranslated region). The miRNA-target genes relationships with scores $\geq 140$ and Energy $\leq-10$, were subjected to further analysis.

The circRNA-miRNA-mRNA network was constructed using Cytoscape software (31) based on the obtained circRNAs, miRNAs and mRNAs.

Clinical samples and cell culture. The present study was approved by the ethics committee of the Lishui Municipal Central Hospital (Lishui, China) and written informed consent was obtained from all patients included in this study. A total of 37 pairs of HCC and adjacent normal tissues without preoperative treatment were collected from surgical resections in the hospital between March 2018 and August 2018 and 
Table II. Primer sequences used for RT-qPCR.

\begin{tabular}{ll}
\hline Gene & \multicolumn{1}{c}{ Sequence $\left(5^{\prime} \rightarrow 3^{\prime}\right)$} \\
\hline$h s a \_c i r c R N A \_100084$ & F: AGAATGCAGGTCCAACCA \\
& R: GAGACAGCGGGAGTGAAG \\
& RT:GTCGTATCCAGTGCAGGGTCCGAGGTATTCGCACTGGATACGCAAATCC \\
& F: GGGGTTCCTGGGGATG \\
& R: GTGCAGGGTCCGAGGT \\
& F: GTCGGCCCAAACCGAG \\
RF 2 R: CGAAGGCCAAGAAGGTGAGAA & F: CTCGCTTCGGCAGCACA \\
R6 & R: AACGCTTCACGAATTTGCGT \\
& F: AGAAGGCTGGGGCTCATTTG \\
RAPDH $:$ AGGGGCCATCCACAGTCTTC
\end{tabular}

RT-qPCR, reverse transcription-quantitative PCR; RT, reverse transcription primer; IGF2, insulin-like growth factor 2.

stored at $-80^{\circ} \mathrm{C}$ until use. $\mathrm{HCC}$ was diagnosed via histological confirmation. The adjacent normal tissues were collected $3 \mathrm{~cm}$ away from the HCC tissue edge. The clinical characteristics of patients are presented in Table I.

Human liver cancer cell lines with a stepwise metastatic potential, including $\mathrm{MHCC} 97 \mathrm{H}$ with high metastatic potential, and HepG2 (a hepatoblastoma cell line) (32) and Hep3B with very low invasiveness, and the normal human hepatic stellate cell LX2 were purchased from The Cell Bank of Type Culture Collection of the Chinese Academy of Sciences and iCell Bioscience Inc. All cells were authenticated via STR profiling. Cells were cultured in Dulbecco's modified Eagle's medium (DMEM; Invitrogen; Thermo Fisher Scientific, Inc.) with $10 \%$ fetal bovine serum (FBS, Invitrogen; Thermo Fisher Scientific, Inc.), $100 \mathrm{U} / \mathrm{ml}$ penicillin, and $100 \mu \mathrm{g} / \mathrm{ml}$ streptomycin (Invitrogen; Thermo Fisher Scientific, Inc.) in a humidified atmosphere of $5 \% \mathrm{CO}_{2}$ at $37^{\circ} \mathrm{C}$.

RNA extraction and reverse transcription-quantitative $(R T-q) P C R$. Total RNA was extracted from tissue and cells $\left(1 \times 10^{6}\right)$ with TRIzol ${ }^{\circledR}$ reagent (Invitrogen; Thermo Fisher Scientific, Inc.), according to the manufacturer's instructions. The quantity and concentration of total RNA were determined by a NanoDrop 2000 instrument (Thermo Fisher Scientific, Inc.). RT-qPCR was performed as described previously (33). Briefly, total RNA was reverse transcribed to cDNA using a PrimeScript RT Reagent kit (Takara Biotechnology Co., Ltd.). qPCR was performed in a 96-well plate on an ABI 7500 system (Applied Biosystems; Thermo Fisher Scientific, Inc.) with PowerUp SYBR-Green Master Mix (Thermo Fisher Scientific, Inc.) as per the procedure provided by the manufacturer. For detecting hsa-miR-23a-5p, a hsa-miR-23a-5p-specific stem-loop primer (Guangzhou RiboBio Co., Ltd.) was used for reverse transcription and RT-qPCR amplification was performed using the Bulge-Loop miRNA RT-qPCR Starter kit (Guangzhou RiboBio Co., Ltd.). The thermocycling conditions were $95^{\circ} \mathrm{C}$ for $10 \mathrm{~min}$, followed by 40 cycles of $95^{\circ} \mathrm{C}$ for $15 \mathrm{sec}$ and $60^{\circ} \mathrm{C}$ for $60 \mathrm{sec}$. GAPDH (for circRNA and mRNA) or U6 (for miRNA) was used as reference control. Relative expression level was calculated by the $2^{-\Delta \Delta \mathrm{Cq}}$ method (34). Primer sequences are listed in Table II.

Western blot analysis. The protein expression of IGF2 was determined by western blotting. Briefly, total protein was extracted using RIPA lysis buffer and protein concentration was determined by BCA assay (Beyotime Institute of Biotechnology). Proteins (30 $\mu \mathrm{g} /$ lane) were separated by SDS-PAGE on $12 \%$ gel and transferred onto a PVDF membrane (Millipore). Following blocking with 5\% skimmed milk for $1 \mathrm{~h}$ at room temperature $\left(\sim 25^{\circ} \mathrm{C}\right)$, the PVDF membrane was incubated with primary antibodies against IGF2 (1:1,000, cat. no. ab9574; Abcam) and GAPDH (1:1,000, cat. no. ab8245; Abcam)_at $4^{\circ} \mathrm{C}$ overnight. Following washing with Tris-buffered saline containing $0.05 \%$ Tween-20, horseradish peroxidase-conjugated secondary antibody $\operatorname{IgG}(\mathrm{H} \& \mathrm{~L}$, 1:4,000, cat. no. ab6728; Abcam) was added and incubated at room temperature $\left(25^{\circ} \mathrm{C}\right)$ for $1 \mathrm{~h}$. Protein bands were visualized using the enhanced chemiluminescence ECL method (Bio-Rad Laboratories, Inc.) and analyzed with ImageJ software v.1.6.0 (National Institutes of Health).

Cell transfection. Sh-hsa_circ_100084 (5'-AACCCGUUC UCCGAAUUCCUAdTdT-3'), hsa-miR-23a-5p mimics, the miRNA negative control (NC), pcDNA3.1-IGF2, and pcDNA3.1-NC were obtained from Guangzhou RiboBio Co., Ltd. These constructs were transfected into HepG2 cells using Lipofectamine ${ }^{\circledR} 2000$ (Invitrogen; Thermo Fisher Scientific, Inc.), according to the manufacturer's instructions. Briefly, $5 \times 10^{5}$ cells were seeded in each well of six-well plates. Sh-hsa circ_100084 (1 $\mu \mathrm{g} /$ well $),$ hsa-miR-23a-5p mimics (50 pmol/well), pcDNA3.1-IGF2 (1 $\mu \mathrm{g} /$ well), or NC (1 $\mu \mathrm{g} /$ well for Sh-NC and $50 \mathrm{pmol} /$ well for miR-NC) and Lipofectamine ${ }^{\circledR} 2000$ were added in each well and incubated for $24 \mathrm{~h}$. Transfection efficiency was determined by RT-qPCR after $24 \mathrm{~h}$.

Cell proliferation, migration and invasion assays. Cell proliferation was analyzed by a Cell Counting Kit-8 (CCK-8, 
Table III. Top 10 upregulated circRNAs and top 10 downregulated circRNAs between HCC and normal tissues.

\begin{tabular}{lllll}
\hline ID & P-value & FDR & logFC & Hsa_circRNAs \\
\hline Downregulated circRNAs & & & & \\
ASCRP005278 & $1.73 \times 10^{-10}$ & $4.01 \times 10^{-08}$ & -6.4099 & hsa_circRNA_105031 \\
ASCRP004769 & $3.51 \times 10^{-07}$ & $9.18 \times 10^{-06}$ & -5.69472 & hsa_circRNA_104515 \\
ASCRP000679 & $6.00 \times 10^{-07}$ & $1.30 \times 10^{-05}$ & -5.29939 & hsa_circRNA_100291 \\
ASCRP004845 & $8.86 \times 10^{-07}$ & $1.75 \times 10^{-05}$ & -4.45749 & hsa_circRNA_104592 \\
ASCRP003527 & $1.62 \times 10^{-06}$ & $2.55 \times 10^{-05}$ & -4.41726 & hsa_circRNA_103229 \\
ASCRP001306 & $3.37 \times 10^{-06}$ & $4.20 \times 10^{-05}$ & -4.19757 & hsa_circRNA_100933 \\
ASCRP000678 & $7.84 \times 10^{-06}$ & $7.18 \times 10^{-05}$ & -3.92181 & hsa_circRNA_100290 \\
ASCRP001459 & $2.60 \times 10^{-06}$ & $3.47 \times 10^{-05}$ & -3.89008 & hsa_circRNA_101091 \\
ASCRP003234 & $1.74 \times 10^{-05}$ & $1.19 \times 10^{-04}$ & -3.61817 & hsa_circRNA_102927 \\
ASCRP002498 & $1.69 \times 10^{-05}$ & $1.16 \times 10^{-04}$ & -3.58558 & hsa_circRNA_102166 \\
Upregulated circRNAs & & & \\
ASCRP000474 & $2.74 \times 10^{-04}$ & $8.82 \times 10^{-04}$ & 3.2099071 & \\
ASCRP000343 & $3.35 \times 10^{-08}$ & $2.04 \times 10^{-06}$ & 3.2221957 & hsa_circRNA_100084 \\
ASCRP003740 & $1.65 \times 10^{-06}$ & $2.57 \times 10^{-05}$ & 3.3864621 & hsa_circRNA_001846 \\
ASCRP001647 & $5.29 \times 10^{-05}$ & $2.64 \times 10^{-04}$ & 3.610212 & hsa_circRNA_103442 \\
ASCRP000535 & $1.71 \times 10^{-06}$ & $2.62 \times 10^{-05}$ & 3.6841615 & hsa_circRNA_101287 \\
ASCRP002369 & $3.13 \times 10^{-07}$ & $8.37 \times 10^{-06}$ & 3.766187 & hsa_circRNA_102034 \\
ASCRP001906 & $6.85 \times 10^{-05}$ & $3.12 \times 10^{-04}$ & 3.8742393 & hsa_circRNA_101555 \\
ASCRP002600 & $5.00 \times 10^{-15}$ & $1.74 \times 10^{-11}$ & 4.0273536 & hsa_circRNA_102272 \\
ASCRP002601 & $1.89 \times 10^{-13}$ & $1.64 \times 10^{-10}$ & 4.249804 & hsa_circRNA_102273 \\
ASCRP004099 & $6.85 \times 10^{-08}$ & $3.60 \times 10^{-06}$ & 4.6531421 & hsa_circRNA_103809 \\
\hline
\end{tabular}

FDR, false discovery rate; HCC, hepatocellular carcinoma; circRNA, circular RNAs.

Beyotime Institute of Biotechnology) according to manufacturer's instructions. Briefly, $1 \times 10^{4}$ cells/well were plated on 96-well plates and incubated at $37^{\circ} \mathrm{C}$ in $5 \% \mathrm{CO}_{2}$ for $24 \mathrm{~h}$. After $48 \mathrm{~h}$ of transfection, the cells were incubated for another 24 , 48 and $72 \mathrm{~h}$. Then, $10 \mu \mathrm{l}$ of CCK-8 was added into each well and the absorbance at $450 \mathrm{~nm}$ was measured with an MK3 microplate reader (Thermo Scientific, Inc.).

Cell migration and invasion assays were performed using Transwell assays. For invasion assays, $1 \times 10^{5}$ cells were suspended into $250 \mu \mathrm{l}$ of serum-free DMEM with $0.1 \%$ bovine serum albumin (Invitrogen; Thermo Fisher Scientific, Inc.) and seeded into the upper chamber of a 24-well Transwell insert (pore size: $8 \mu \mathrm{m}$; BD Biosciences) which were precoated with Matrigel at $37^{\circ} \mathrm{C}$ for $30 \mathrm{~min}(\mathrm{BD}$ Biosciences). The lower chamber was filled with DMEM containing $2.5 \%$ FBS. For migration assays, $1 \times 10^{5}$ suspended cells were seeded into the upper chambers without a Matrigel coating. After $48 \mathrm{~h}$, the invaded or migrated cells were fixed and stained with $0.5 \%$ crystal violet at room temperature $\left(\sim 25^{\circ} \mathrm{C}\right)$ for $15 \mathrm{~min}$ and counted under a microscope (Olympus Corporation). Five images were randomly captured for each sample.

Luciferase reporter assay. HepG2 cells were seeded into 24-well plates and co-transfected with hsa_circ_100084-wt, hsa_circ_100084-mut, IGF2-wt, or IGF2-mut plasmids. Then, hsa-miR-23a-5p mimics or negative controls (Guangzhou
RiboBio Co., Ltd.) were transfected into cells using Lipofectamine $^{\circledast} 2000$ (Invitrogen; Thermo Fisher Scientific, Inc.). Luciferase assays were conducted $48 \mathrm{~h}$ following transfection using a Dual Luciferase Reporter Assay System (Promega Corporation). The firefly luciferase activity was normalized to Renilla luciferase activity.

Immunohistochemistry (IHC). The protein level of IGF2 in hepatic tissues was examined by IHC. Briefly, biopsies were fixed in $4 \%$ buffered formaldehyde and embedded in paraffin for $4 \mathrm{~h}$. Then, $5-\mu \mathrm{m}$ sections were blocked with 5\% BSA (cat. no. 810652; Sigma-Aldrich; Merck KGaA) for $30 \mathrm{~min}$ at $37^{\circ} \mathrm{C}$, and incubated with a primary antibody against IGF2 (1:500; cat. no. ab9574, Abcam) overnight at $4^{\circ} \mathrm{C}$, followed by incubation with a biotinylated secondary anti-Rabbit $\operatorname{IgG}$ antibody (1:500; cat. no. SA00004-2, ProteinTech Group, Inc.) and peroxidase-labeled streptavidin at room temperature for $15 \mathrm{~min}$. Representative images at magnification, $\mathrm{x} 20$ and $\mathrm{x} 40$ were captured under an inverted microscope (Leica Microsystems, Inc.).

Statistical analysis. All experiments were performed in triplicate and data were analyzed by SPSS 20.0 (IBM Corp.). Comparisons between two groups were analyzed by Student's t-test or $\chi^{2}$ test when appropriate, while comparisons among multiple groups were conducted using one-way ANOVA with LSD post hoc analysis. $\mathrm{P}<0.05$ was considered to indicate a statistically significant difference. 

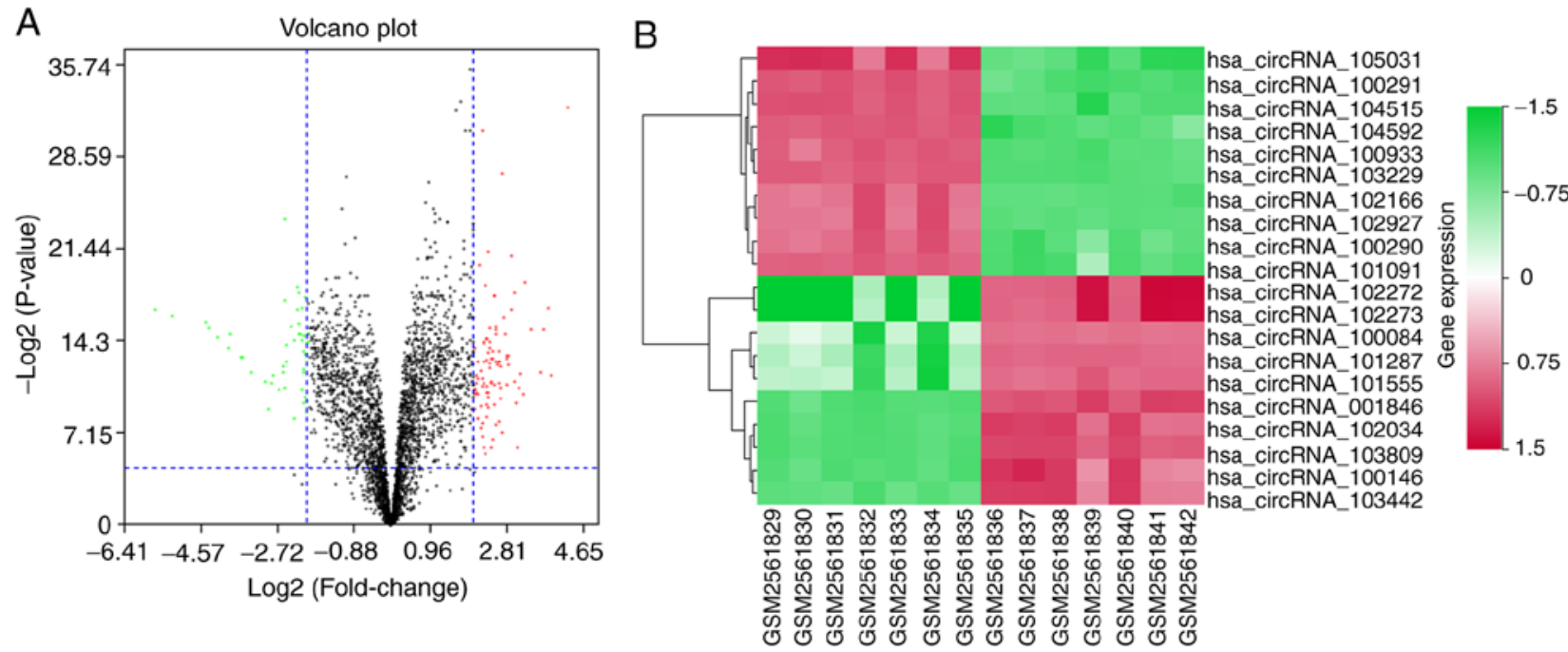

Figure 1. DE-circRNAs in the liver cancer samples compared with the adjacent liver tissues. (A) Volcano plot displaying the DE-circRNAs. (B) Heatmap showing the top 10 upregulated circRNAs and top 10 downregulated circRNAs. DE, differentially expressed; circRNAs, circular RNAs.

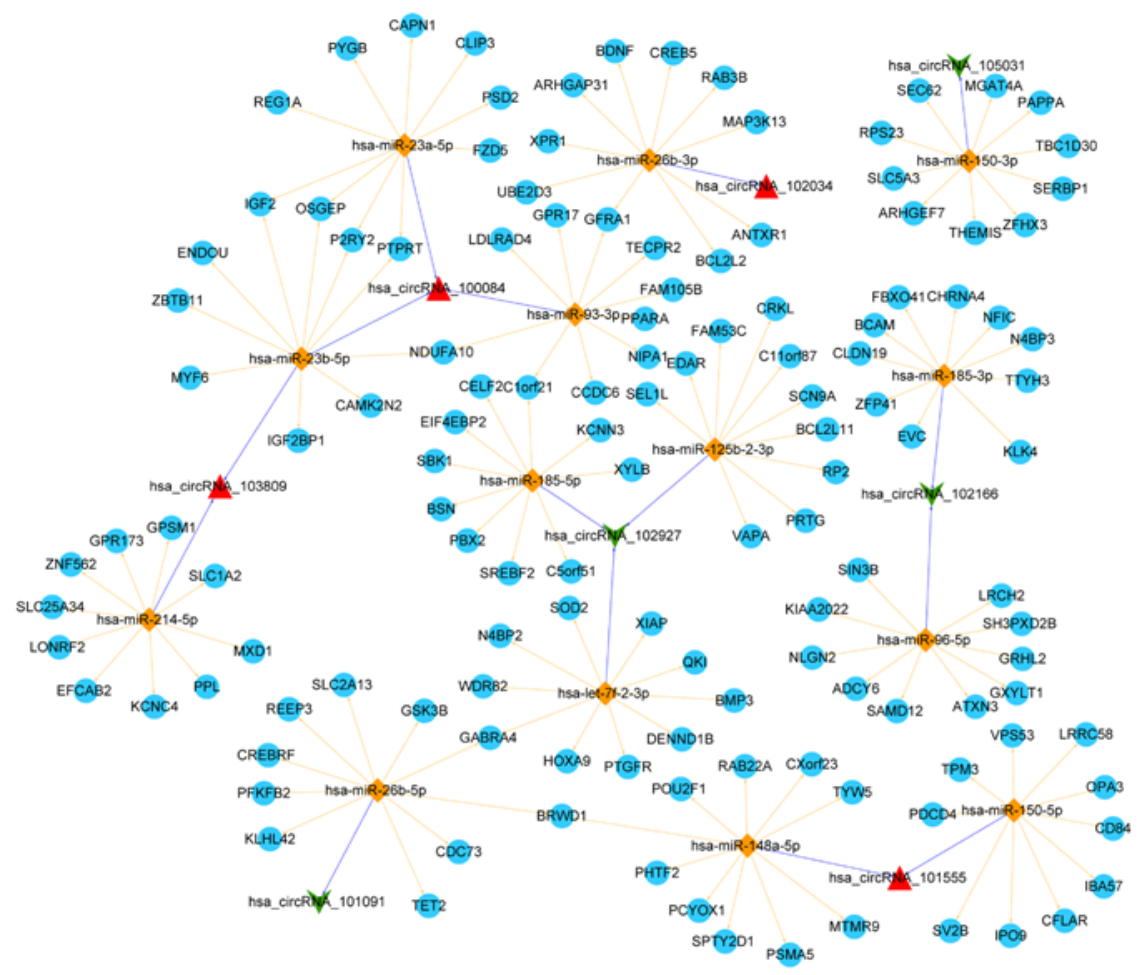

Figure 2. Competitive endogenesis network among differentially expressed circRNAs, miRNAs and target genes. circRNAs, circular RNAs; miRNAs, microRNAs.

\section{Results}

Identification of DE-circRNAs in HCC samples. The circRNA expression profile of HCC deposited by Han et al (25), was downloaded from the GEO database and GEO2R was used to identify DE-circRNAs in the HCC samples compared with the adjacent liver tissues. According to the criteria of FDR $<0.05$ and $\mid \log \mathrm{FCl}>2,147$ DE-circRNAs were identified, including 50 downregulated circRNAs $(34.01 \%)$ and 97 upregulated circRNAs (65.99\%; Fig. 1A). The top 10 upregulated circRNAs and top 10 downregulated circRNAs are shown in Fig. 1B and listed in Table III.
Construction of circRNA-miRNA-mRNA network. By using miRanda to predict which miRNAs were related to the top 20 DE circRNAs, 409 miRNAs were obtained at the criteria of score $\geq 140$ and Energy $\leq-10$. Following searching in miR2 Disease, 17 circRNA-miRNA relationships involving 16 miRNAs and 8 circRNAs were obtained. Additionally, a total of 1,669 target genes associated with these miRNAs were predicted in 6 of 7 searched databases and 923 target genes were further filtered. The top 10 target genes for each miRNA were used for constructing a ceRNA network in Cytoscape. This ceRNA network involved 15 circRNA-miRNA relationships and 140 miRNA-mRNA relationships (Fig. 2). 

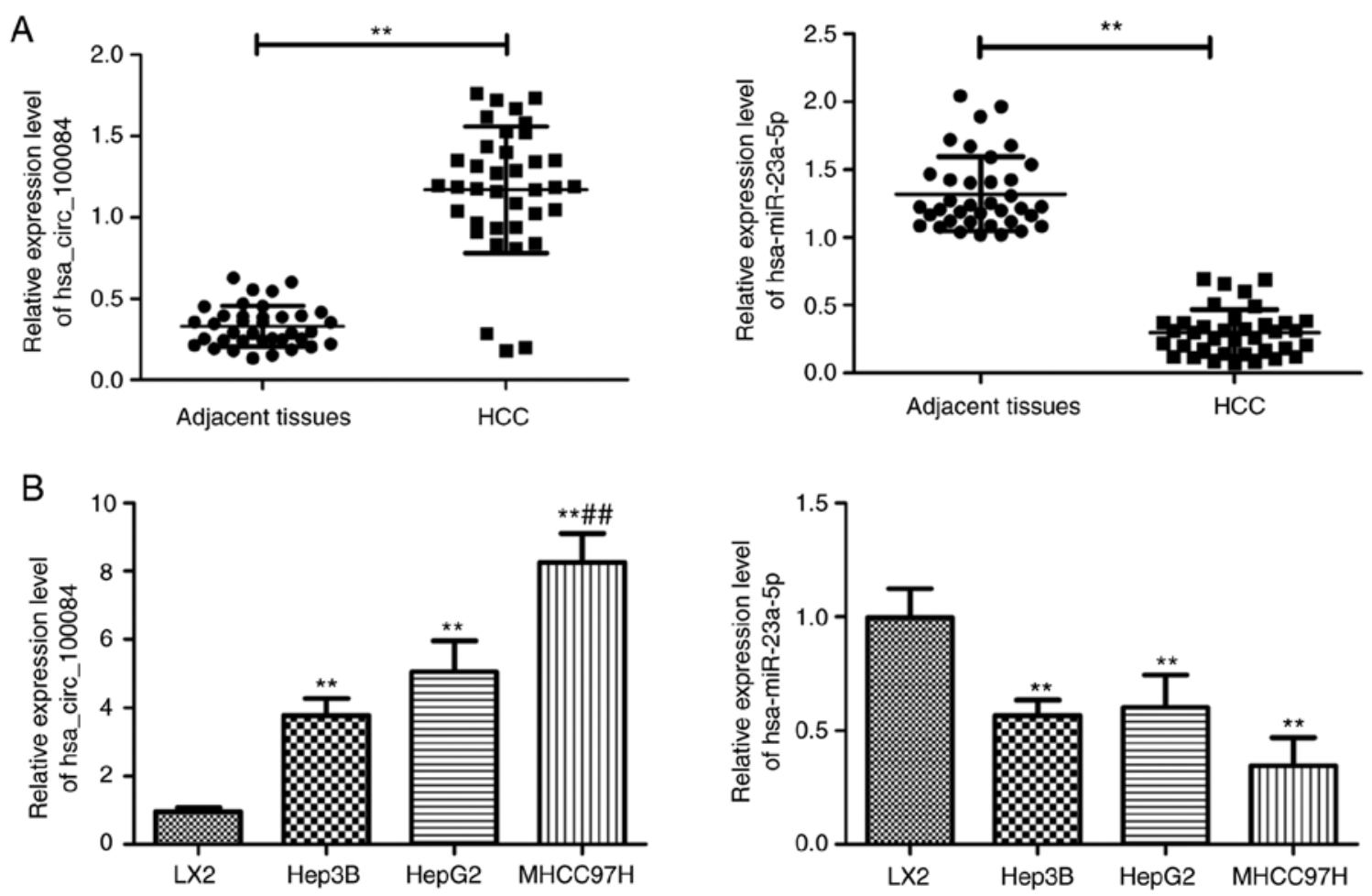

Figure 3. Relative expression levels of hsa_circ_100084 and hsa-miR-23a-5p in HCC tissues and liver cancer cells. (A) Hsa_circ_100084 and hsa-miR-23a-5p expression in 37 pairs of HCC tissues and adjacent non-tumor tissues detected by RT-qPCR. Comparisons between two groups were analyzed by Student's $t$ test. ${ }^{* *} \mathrm{P}<0.01$. (B) The qPCR analysis of hsa_circ_100084_ and hsa-miR-23a-5p for the normal hepatic cell LX2, and for liver cancer cell lines with different metastatic abilities (Hep3B, HepG2 and MHCC97H). Comparisons among multiple groups were conducted using one-way ANOVA with LSD post hoc analysis. ${ }^{* *} \mathrm{P}<0.01$ vs. normal cell line LX2; ${ }^{\# \#} \mathrm{P}<0.01$ vs. Hep3B and HepG2. HCC, hepatocellular carcinoma; RT-qPCR, reverse transcription-quantitative PCR.

Differential expression of hsa_circRNA_100084, hsa-miR-23a-5p, and IGF2 in HCC tissues and cells. Findings showed that IGF2 was involved in the ceRNA relationship of hsa_circRNA_100084-hsa-miR-23a-5p-IGF2. Therefore, the expression level of hsa_circRNA_100084, hsa-miR-23a-5p, and IGF2 in HCC tissues and liver cancer cells we validated by RT-qPCR, IHC and western blotting. As shown in Fig. 3A, compared with levels in the adjacent normal tissues, the relative expression levels of hsa_circRNA_100084 in HCC tissues were significantly upregulated $(\mathrm{P}<0.01)$, while the expression of hsa-miR-23a-5p was significantly downregulated $(\mathrm{P}<0.01)$. Then, the expression patterns of hsa_circRNA_100084 and hsa-miR-23a-5p were analyzed in three liver cancer cell lines with different metastatic potential, including $\mathrm{MHCC} 97 \mathrm{H}$, Hep3B and HepG2 cells. Consistently, the expression of hsa_circRNA_100084 was significantly upregulated in liver cancer cells (Hep3B and MHCC97H) and a hepatoblastoma cell line $(\mathrm{HepG} 2)(\mathrm{P}<0.01)$, while the expression of hsa-miR-23a-5p was significantly downregulated compared with levels in the human normal hepatic cell line LX2 $(\mathrm{P}<0.01$, Fig. 3B). Additionally, the mRNA and protein expression of IGF2 in HCC tissues and adjacent tissues were tested by RT-qPCR, IHC and western blotting (Fig. 4). IHC results showed that the number of IGF2-positive cells in HCC tissues was much higher compared with adjacent normal tissues (Fig. 4A and B). qPCR and western blotting further confirmed that IGF2 was upregulated in HCC tissues and cells (Fig. 4C-E). Taken together, these data suggested that hsa-miR-23a-5p, hsa_circRNA_100084 and IGF2 might be involved in HCC progression and that there may be competing relationships among them.

hsa_circRNA_100084 promotes IGF2 expression by acting as a sponge of hsa-miR-23a-5p in liver cancer cells. RT-qPCR results showed that expression of hsa_circRNA_100084 was significantly decreased following the transfection of sh-hsa_circRNA_100084, while hsa-miR-23a-5p expression was significantly increased following the transfection of hsa-miR-23a-5p mimics ( $\mathrm{P}<0.01$; Fig. 5A). Bioinformatics analysis demonstrated that hsa_circRNA_100084 and IGF2 may bind to hsa-miR-23a-5p (Fig. 5B). Luciferase reporter assays showed that hsa-miR-23a-5p mimics could regulate the luciferase activity of wild-type hsa_circRNA_100084 and IGF2 $(\mathrm{P}<0.01$; Fig. 5C), rather than mutant hsa_circRNA_100084 and IGF2 ( $>0.05$; Fig. 5C). To further investigate the relationships among hsa_circRNA_100084, hsa-miR-23a-5p and IGF2, HepG2 cells were transfected with sh-hsa_circRNA_100084, hsa-miR-23a-5p, mimics or sh-hsa_circRNA_100084 + hsa-miR-23a-5p mimics, respectively. RT-qPCR results showed that sh-hsa_circRNA_100084 transfection increased the levels of hsa-miR-23a-5p (P<0.01; Fig. 5D). Additionally, overexpression of hsa-miR-23a-5p decreased the expression of IGF2 in HepG2 cells. However, sh-hsa_circRNA_100084 could also attenuate the effect of hsa-miR-23a-5p overexpression on the expression of IGF2 ( $\mathrm{P}<0.01$; Fig. 5D). Taken together, the results of the present study demonstrated that hsa_circRNA_100084 promoted the expression of $I G F 2$ by acting as a sponge of hsa-miR-23a-5p in liver cancer cells. 
A

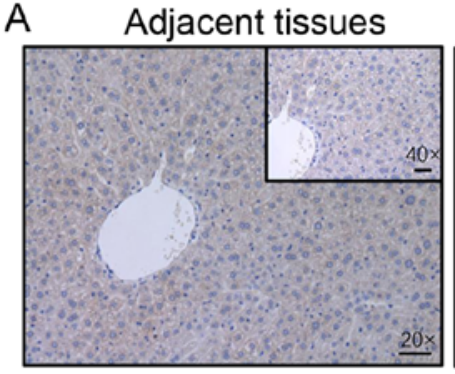

C

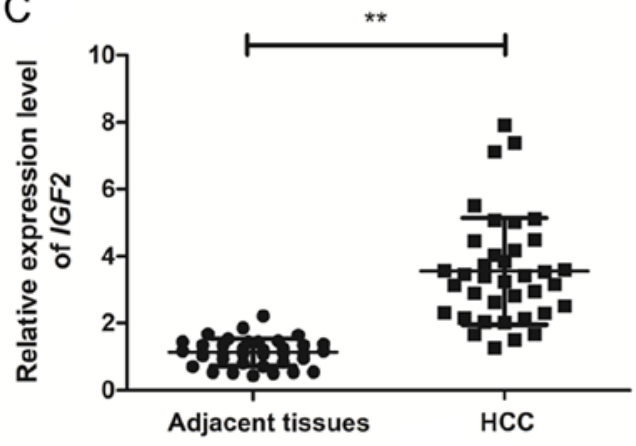

E

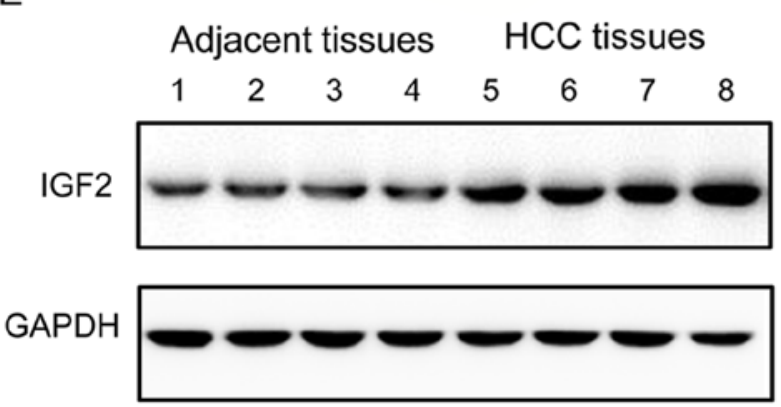

HCC tissues

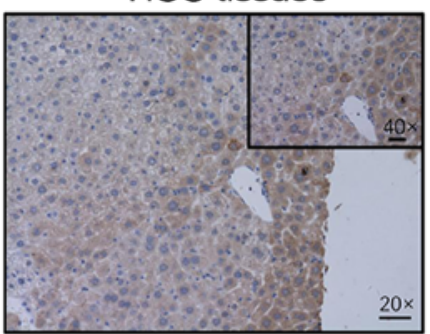

B

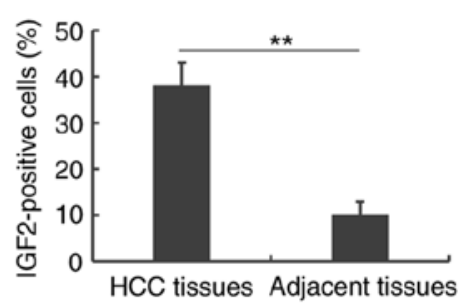

D

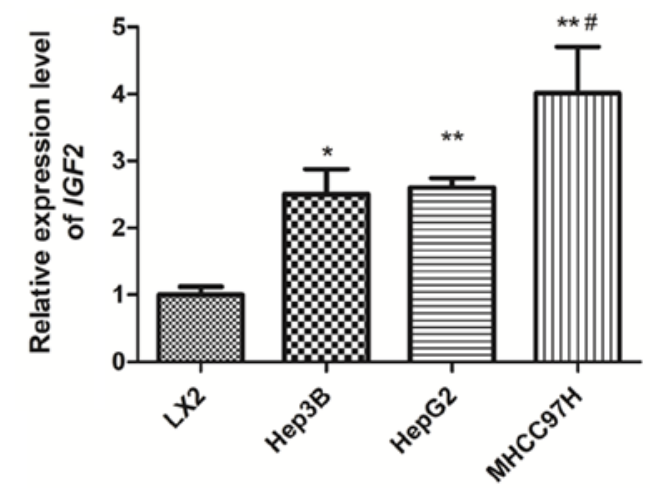

$\mathrm{F}$

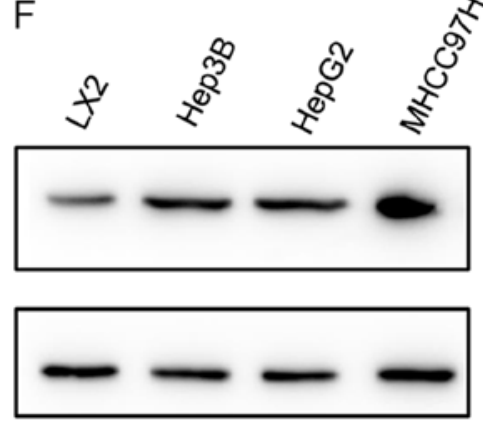

Figure 4. Expression of IGF2 in HCC tissues and liver cancer cell lines. (A) Representative images for IGF2 immunohistochemistry staining in human HCC samples. (B) The percentage of IGF2-positive cells in HCC and adjacent tissues. The presented values are the means \pm standard deviation from five different images (magnification, $\mathrm{x} 40$ ). Comparisons between two groups were analyzed by Student's $t$ test. ${ }^{* *} \mathrm{P}<0.01$. (C) IGF2 expression in 37 pairs of HCC tissues and adjacent non-tumor tissues detected by RT-qPCR. Comparisons between two groups were analyzed by Student's t test. ${ }^{* *} \mathrm{P}<0.01$. (D) The qPCR analysis of IGF2 expression in a normal hepatic cell LX2 and liver cancer cell lines. Comparisons among multiple groups were conducted using one-way ANOVA with LSD post hoc analysis. ${ }^{*} \mathrm{P}<0.05,{ }^{* *} \mathrm{P}<0.01$ vs. normal cell line LX2; ${ }^{*} \mathrm{P}<0.05$ vs. Hep3B and HepG2. (E) The western blot analysis of IGF2 in 4 pairs of HCC tissues and adjacent non-tumor tissues. (F) The western blot analysis of IGF2 in a normal hepatic cell LX2 and liver cancer cell lines. GAPDH was used as internal control. IGF2, insulin-like growth factor 2; HCC, hepatocellular carcinoma; RT-qPCR, reverse transcription-quantitative PCR.

Hsa_circRNA_100084 promotes proliferation, migration and invasion via regulating IGF2. CCK-8 assays and Transwell assays were performed to investigate the role of hsa_circRNA_100084 on cell proliferation, migration and invasion using HepG2 cells. The results demonstrated that sh-hsa_circRNA_100084 inhibited the proliferation, migration and invasion of liver cancer cells. However, transfection of pcDNA3.1-IGF2 simultaneously could reverse the effects of sh-hsa_circRNA_100084 (Fig. 6). Taken together, the results suggested that hsa_circRNA_100084 promotes liver cancer cell proliferation, migration and invasion by regulating IGF2 via acting as a sponge of hsa-miR-23a-5p.

\section{Discussion}

HCC is regarded as the most malignant type of liver cancer because of its high incidence rate and poor prognosis (35).
Therefore, it is necessary to investigate the biological basis and identify novel targets for HCC. Due to their special structure, circRNAs are evolutionarily conserved and stable. Previous studies have demonstrated that circRNAs are disease-, tissue- and stage-specifically expressed, suggesting their particular roles in disease initiation and development (36-38). The present study re-analyzed the gene expression profile GSE97332 and identified 147 DE-circRNAs, including 50 downregulated circRNAs $(34.01 \%)$ and 97 upregulated circRNAs (65.99\%). Then, a ceRNA network was constructed for these DE-circRNAs. In this ceRNA network, it was found that $I G F 2$ was involved in a ceRNA relationship of hsa_circ RNA_100084-hsa-miR-23a-5p-IGF2. Further studies demonstrated that hsa_circRNA_100084 promoted liver cancer cell proliferation, migration and invasion by competitively binding hsa-miR-23a-5p, leading to the upregulation of $I G F 2$. 
A

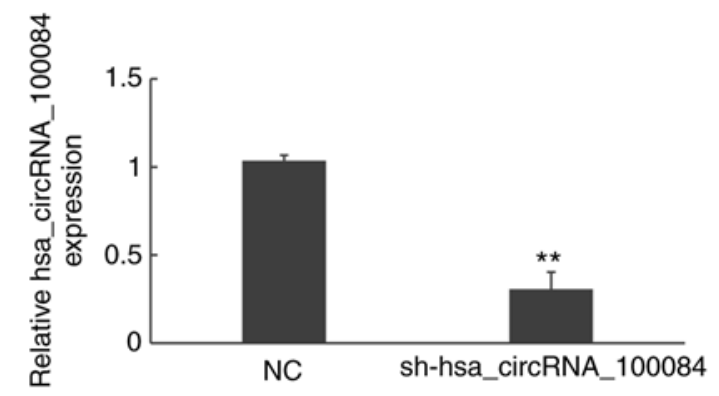

B hsa_circRNA_100084-WT: ACAUAGAGAAAUUAGGAACCCC... hsa-miR-23a-5p: UUAGGGUAGGGUUCCUUGGGG IGF2 3'UTR-WT: TGGCAAGACAGCCGGGAACCCCT hsa_circRNA_100084-mut: ACAUAGAGAAAUUACCAUGCCC...

IGF2 3'UTR-mut:TGGCAAGACAGCCGCCAUGCCCT

$\mathrm{D} \div$

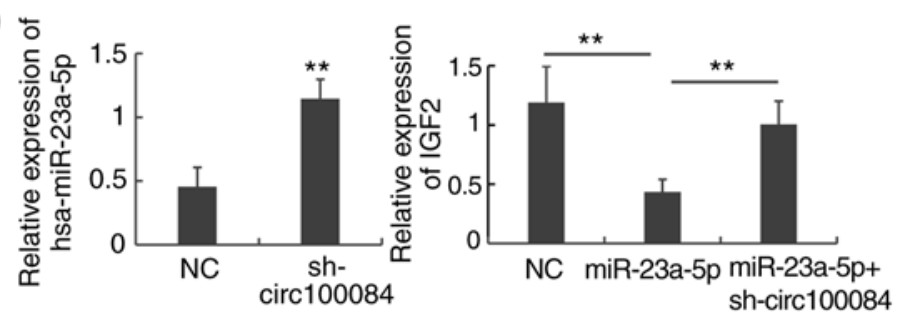

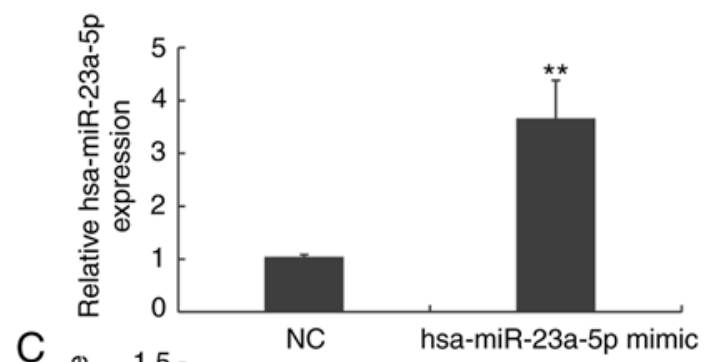
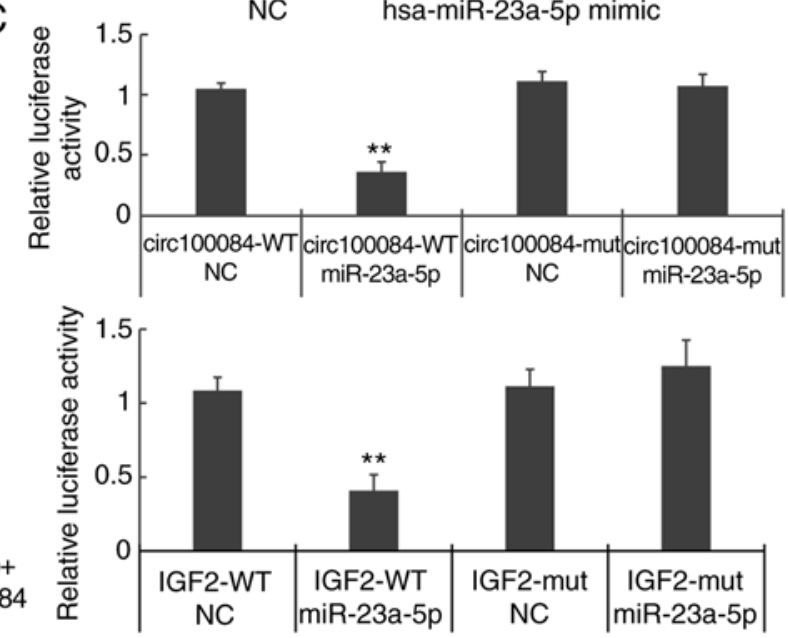

Figure 5. Relationships among hsa_circ_100084, hsa-miR-23a-5p and IGF2. (A) Relative expression of hsa_circ_100084 and hsa-miR-23a-5p following transfection with sh-hsa_circ_100084 or hsa-miR-23a-5p mimics. (B) Putative binding sites among hsa_circ_100084, hsa-miR-23a-5p and IGF2. (C) The luciferase activity of LUC-circ100084-WT, LUC-IGF2-WT, LUC-circ100084-mutant, or LUC-IGF2-mutant co-transfected with hsa-miR-23a-5p mimics. (D) Relative expression of hsa-miR-23a-5p and IGF2 following transfection with sh-hsa_circ_100084 or co-transfection of hsa-miR-23a-5p mimics and sh-hsa_circ_100084. All data are representative of three independent experiments and expressed as mean \pm standard deviation. Comparisons between two groups were analyzed by Student's t test, while comparisons among multiple groups were conducted using one-way ANOVA with LSD post hoc analysis. ${ }^{* *} \mathrm{P}<0.01$ vs. NC.

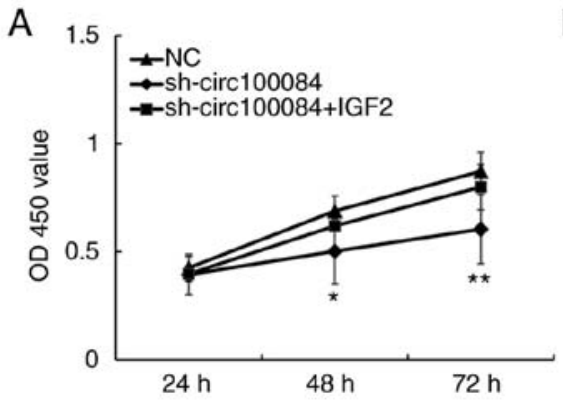

B
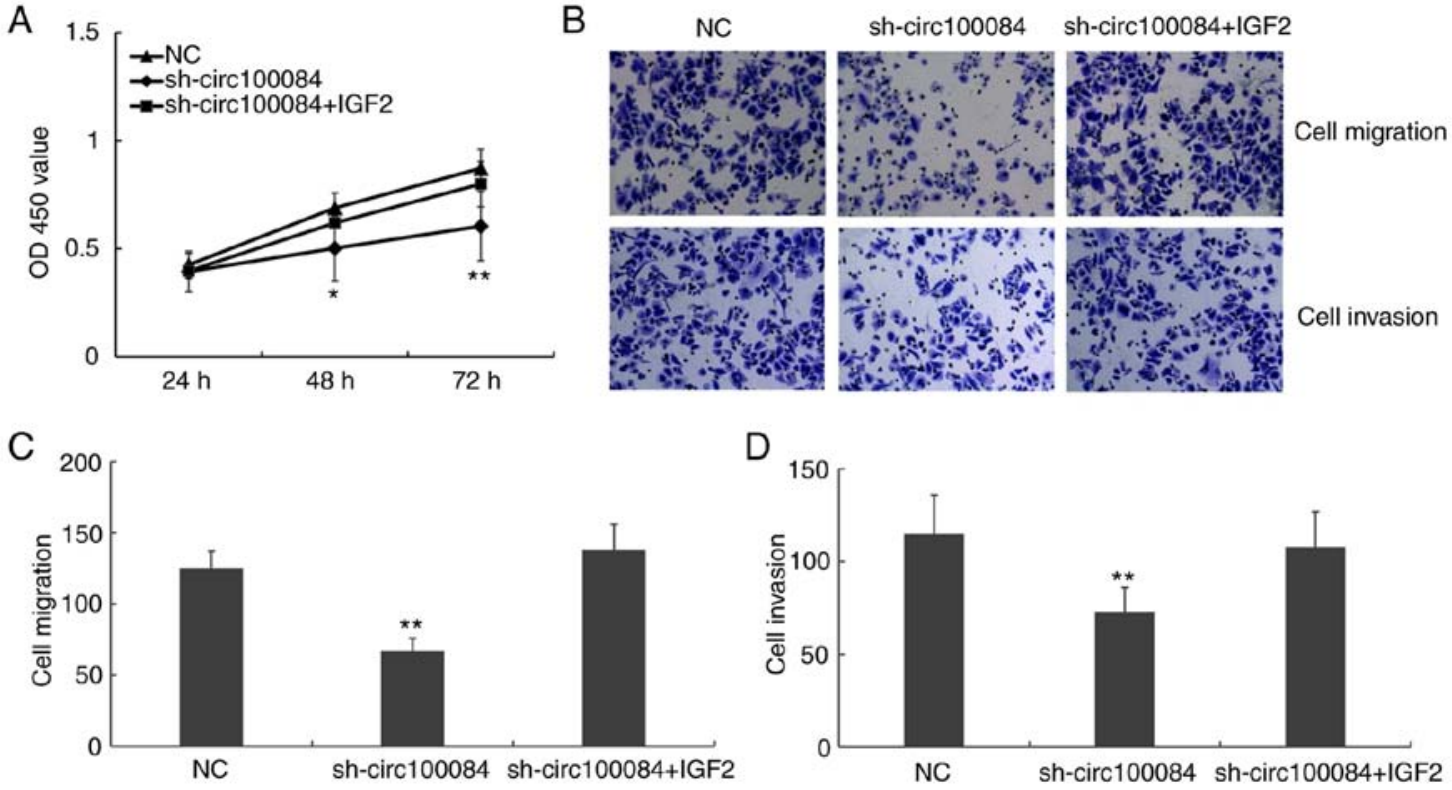

Figure 6. Hsa_circ_100084 regulates liver cancer cell proliferation, migration and invasion by modulating IGF2. (A) CCK-8 assay was used to measure cell proliferation in HepG2 cells. (B-D) Transwell assays determined cell migration and invasion in HepG2 cells. Magnification, x200. All data are representative of three independent experiments and expressed as mean \pm standard deviation. Comparisons between two groups were analyzed by Student's $t$ test, while comparisons among multiple groups were conducted using one-way ANOVA with LSD post hoc analysis. ${ }^{* *} \mathrm{P}<0.01$ vs. NC.

Elucidating the molecular mechanisms of $\mathrm{HCC}$ will be important for the development of therapies to successfully treat HCC. Several studies have demonstrated that IGF2 is upregulated in a number of cancers, including HCC, and is associated with resistance to chemotherapy and a worse prognosis $(18,19,39)$. Though loss of imprinting, loss of hetero- 
zygosity, or reactivation of $I G F 2$ transcription could partially explain the upregulation of $I G F 2$ in cancer, further studies are necessary to explore these possibilities. The present study found that $I G F 2$ is involved in a ceRNA relationship of hsa_circRNA_100084-hsa-miR-23a-5p-IGF2. Consistently, Zhen et al (40) demonstrated that circHMGCS1 promotes hepatoblastoma cell proliferation by regulating $I G F 2$.

The results of the present study demonstrated that knocking down hsa_circRNA_100084 could significantly inhibit the proliferation, migration and invasion of liver cancer cells, suggesting that hsa_circRNA_100084 might have potential to be used us a promising therapeutic strategy for HCC. By searching circBase, hsa_circRNA_100084 was found to correspond to circEIF4G3, which is located on chr1:21329205-21415706. However, the role of this circRNA in cancer initiation and development has not been investigated previously.

The mechanisms of circRNAs in cancer initiation and progression have not been clearly elucidated. It has been reported that circRNAs can regulate the expression of oncogenes or tumor-suppressive genes in different patterns (41). The most reported pattern is the ceRNA hypothesis. In this hypothesis, circRNAs have been proposed to communicate with mRNAs by competing for binding to shared miRNA targets (42). This hypothesis has been confirmed in a number of studies. For example, circMTO1 suppresses HCC progression by acting as a sponge of miR-9 (25). circSMAD2 can inhibit the epithelial-mesenchymal transition via targeting miR-629 in HCC (43). circ_0067934 promotes tumor metastasis and growth in HCC via the inhibition of miR-1324 (44). It was hypothesized that hsa_circRNA_100084 might act as a miRNA sponge. Therefore, it was predicted the miRNAs related with DE-circRNAs by bioinformatics analysis. The combination of hsa-miR-23a-5p with hsa_circRNA_100084, as well as IGF2, was validated by a dual-luciferase reporter assay. As expected, hsa-miR-23a-5p could diminish the fluorescence of the wildtype of hsa_circRNA_100084 and IGF2, but not the mutated forms. In addition, overexpression of hsa-miR-23a-5p could decrease the expression of IGF2 in HepG2 cells. However, sh-hsa_circRNA_100084 at the same time could attenuate the effect of hsa-miR-23a-5p overexpression on the expression of $I G F 2$.

However, the present study inevitably possess some limitations. First, although a ceRNA relationship of hsa_circRNA_100084-hsa-miR-23a-5p-IGF2 axis was identified and their relationship confirmed by experiments, the expression downstream of IGF-2, such as the insulin receptor substrate $1 / \mathrm{PI} 3 \mathrm{~K} / \mathrm{Akt}$ axis and sarcomatoid hepatocellular carcinoma/growth factor receptor-bound protein 2/Ras/mitogen-activated protein kinase axis has not been examined. Besides, more direct evidence, such as RNA immunoprecipitation analysis of the interaction of circRNA_100084 and miR-23a-5p was not performed. Therefore, further researches are still needed to make more validate conclusions.

In conclusion, the present study demonstrated that hsa circRNA_100084 is upregulated in HCC tissue compared with the matched non-tumor liver tissues and may act as a ceRNA to increase $I G F 2$ expression by sponging hsa-miR-23a-5p, which consequently contributes to HCC proliferation, migra- tion and invasion. The deregulated circRNAs in $\mathrm{HCC}$ will be the subject of continuing investigation in further studies.

\section{Acknowledgements}

Not applicable.

\section{Funding}

No funding was received.

\section{Availability of data and materials}

The datasets used during the present study are available from the corresponding author on reasonable request.

\section{Authors' contributions}

JY and YHW designed the study. YL and JL analyzed and interpreted the RNA sequencing data and patients' data. JY, ZCY, YFZ and JFT performed the in vitro experiments, and JY and YHW were major contributors in writing the manuscript. All authors read and approved the final manuscript.

\section{Ethics approval and consent to participate}

This study was approved by the ethics committee of the Lishui Municipal Central Hospital and written informed consent was obtained from all patients included in this study.

\section{Patient consent for publication}

Not applicable.

\section{Competing interests}

The authors declare that they have no competing interests.

\section{References}

1. Forner A, Llovet JM and Bruix J: Hepatocellular carcinoma. Lancet 379: 1245-1255, 2012.

2. Torre LA, Bray F, Siegel RL, Ferlay J, Lortet-Tieulent J and Jemal A: Global cancer statistics, 2012. CA Cancer J Clin 65: 87-108, 2015

3. Lee JI, Kim JK, Kim DY, Ahn SH, Park JY, Kim SU, Kim BK, Han KH and Lee KS: Prognosis of hepatocellular carcinoma patients with extrahepatic metastasis and the controllability of intrahepatic lesions. Clin Exp Metastasis 31: 475-482, 2014.

4. Ferlay J, Soerjomataram I, Dikshit R, Eser S, Mathers C, Rebelo M, Parkin DM, Forman D and Bray F: Cancer incidence and mortality worldwide: Sources, methods and major patterns in GLOBOCAN 2012. Int J Cancer 136: E359-E386, 2015.

5. Nigro JM, Cho KR, Fearon ER, Kern SE, Ruppert JM, Oliner JD, Kinzler KW and Vogelstein B: Scrambled exons. Cell 64: 607-613, 1991.

6. Chen LL: The biogenesis and emerging roles of circular RNAs. Nat Rev Mol Cell Biol 17: 205-211, 2016.

7. Meng S, Zhou H, Feng Z, Xu Z, Tang Y, Li P and Wu M: CircRNA: Functions and properties of a novel potential biomarker for cancer. Mol Cancer 16: 94, 2017.

8. Zhang HD, Jiang LH, Sun DW, Hou JC and Ji ZL: CircRNA: A novel type of biomarker for cancer. Breast Cancer 25: 1-7, 2018.

9. Cui S, Qian Z, Chen Y, Li L, Li P and Ding H: Screening of upand downregulation of circRNAs in HBV-related hepatocellular carcinoma by microarray. Oncol Lett 15: 423-432, 2018. 
10. Kou P, Zhang C, Lin J and Wang H: Circular RNA hsa circ_0078602 may have potential as a prognostic biomarker for patients with hepatocellular carcinoma. Oncol Lett 17: 2091-2098, 2019.

11. Nakamura M, Chiba T, Kanayama K, Hiroaki Kanzaki H, Saito T, Kusakabe Y and Kato N: Epigenetic dysregulation in hepatocellular carcinoma: An up-to-date review. Hepatol Res 49: 3-13, 2019.

12. Lv Y, Wei W, Huang Z, Chen Z, Fang Y, Pan L, Han X and Xu Z: Long non-coding RNA expression profile can predict early recurrence in hepatocellular carcinoma after curative resection. Hepatol Res 48: 1140-1148, 2018.

13. Ma Y, Zhang C, Zhang B, Yu H and Yu Q: circRNA of AR-suppressed PABPC1 $91 \mathrm{bp}$ enhances the cytotoxicity of natural killer cells against hepatocellular carcinoma via upregulating UL16 binding protein 1. Oncol Lett 17: 388-397, 2019.

14. Xie B, Zhao Z, Liu Q, Wang X, Ma Z and Li H: CircRNA has circ_0078710 acts as the sponge of microRNA-31 involved in hepatocellular carcinoma progression. Gene 683: 253-261, 2019.

15. Huang XY, Huang ZL, Zhang PB, Huang XY, Huang J, Wang HC, $\mathrm{Xu}$ B, Zhou J and Tang ZY: CircRNA-100338 is associated with mTOR signaling pathway and poor prognosis in hepatocellular carcinoma. Front Oncol 9: 392, 2019.

16. Hsu CM, Lin PM, Lin HC, Lai CC, Yang CH, Lin SF and Yang MY: Altered expression of imprinted genes in squamous cell carcinoma of the head and neck. Anticancer Res 36: 2251-2258, 2016.

17. $\mathrm{Vu} \mathrm{TH}$ and Hoffman AR: Promoter-specific imprinting of the human insulin-like growth factor-II gene. Nature 371: 714-717, 1994

18. Livingstone C: IGF2 and cancer. Endocr Relat Cancer 20 R321-R339, 2013.

19. Brouwer-Visser J and Huang GS: IGF2 signaling and regulation in cancer. Cytokine Growth Factor Rev 26: 371-377, 2015.

20. Tovar V, Alsinet C, Villanueva A, Hoshida Y, Chiang DY, Solé M, Thung S, Moyano S, Toffanin S, Mínguez B, et al: IGF activation in a molecular subclass of hepatocellular carcinoma and pre-clinical efficacy of IGF-1R blockage. J Hepatol 52: 550-559, 2010.

21. Martinez-Quetglas I, Pinyol R, Dauch D, Torrecilla S, Tovar V, Moeini A, Alsinet C, Portela A, Rodriguez-Carunchio L, Solé M, et al: IGF2 is up-regulated by epigenetic mechanisms in hepatocellular carcinomas and is an actionable oncogene product in experimental models. Gastroenterology 151: 1192-1205, 2016.

22. Yang C, Wu D, Gao L, Liu X, Jin Y, Wang D, Wang T and Li X: Competing endogenous RNA networks in human cancer: Hypothesis, validation, and perspectives. Oncotarget 7: 13479-13490, 2016.

23. Qi X, Zhang DH, Wu N, Xiao JH, Wang X and Ma W: ceRNA in cancer: Possible functions and clinical implications. J Med Genet 52: 710-718, 2015.

24. Du H and Chen Y: Competing endogenous RNA networks in cervical cancer: Function, mechanism, and perspective. J Drug Target 27: 1-47, 2018.

25. Han D, Li J, Wang H, Su X, Hou J, Gu Y, Qian C, Lin Y, Liu X, Huang M, et al: Circular RNA circMTO1 acts as the sponge of microRNA-9 to suppress hepatocellular carcinoma progression. Hepatology 66: 1151-1164, 2017.

26. Barrett T, Wilhite SE, Ledoux P, Evangelista C, Kim IF, Tomashevsky M, Marshall KA, Phillippy KH, Sherman PM, Holko M, et al: NCBI GEO: Archive for functional genomics data sets--update. Nucleic Acids Res 41 (D1): D991-D995, 2013.

27. Ritchie ME,Phipson B, Wu D, Hu Y,Law CW, Shi W and Smyth GK: limma powers differential expression analyses for RNA-sequencing and microarray studies. Nucleic Acids Res 43: e47, 2015.

28. Smyth GK: Linear models and empirical bayes methods for assessing differential expression in microarray experiments. Stat Appl Genet Mol Biol 3: Article3, 2004.
29. Turner DA: Miranda: A non-strict functional language with polymorphic types. In: Proc. of a Conference on Functional Programming Languages and Computer Architecture. Jouannaud JP (ed). SpringerVerlag, Berlin, Heidelberg, pp 1-16, 1985.

30. Jiang Q, Wang Y, Hao Y, Juan L, Teng M, Zhang X, Li M, Wang $\mathrm{G}$ and Liu Y: miR2Disease: A manually curated database for microRNA deregulation in human disease. Nucleic Acids Res 37 (Database): D98-D104, 2009.

31. Shannon P, Markiel A, Ozier O, Baliga NS, Wang JT, Ramage D, Amin N, Schwikowski B and Ideker T: Cytoscape: A software environment for integrated models of biomolecular interaction networks. Genome Res 13: 2498-2504, 2003.

32. López-Terrada D, Cheung SW, Finegold MJ and Knowles BB: Hep G2 is a hepatoblastoma-derived cell line. Hum Pathol 40: 1512-1515, 2009.

33. Xu D, Yu J, Gao G, Lu G, Zhang Y and Ma P: LncRNA DANCR functions as a competing endogenous RNA to regulate RAB1A expression by sponging miR-634 in glioma. Biosci Rep 38: BSR20171664, 2018

34. Livak KJ and Schmittgen TD: Analysis of relative gene expression data using real-time quantitative PCR and the 2(-Delta Delta C(T)) Method. Methods 25: 402-408, 2001.

35. Zhu RX, Seto WK, Lai CL and Yuen MF: Epidemiology of hepatocellular carcinoma in the Asia-Pacific region. Gut Liver 10: 332-339, 2016.

36. Salzman J, Chen RE, Olsen MN, Wang PL and Brown PO: Cell-type specific features of circular RNA expression. PLoS Genet 9: e1003777, 2013

37. Memczak S, Jens M, Elefsinioti A, Torti F, Krueger J, Rybak A, Maier L, Mackowiak SD, Gregersen LH, Munschauer M, et al: Circular RNAs are a large class of animal RNAs with regulatory potency. Nature 495: 333-338, 2013.

38. Salzman J, Gawad C, Wang PL, Lacayo N and Brown PO: Circular RNAs are the predominant transcript isoform from hundreds of human genes in diverse cell types. PLoS One 7: e30733, 2012.

39. Zatkova A, Rouillard JM, Hartmann W, Lamb BJ, Kuick R, Eckart M, von Schweinitz D, Koch A, Fonatsch C, Pietsch T, et al: Amplification and overexpression of the IGF2 regulator PLAG1 in hepatoblastoma. Genes Chromosomes Cancer 39: 126-137, 2004.

40. Zhen N, Gu S, Ma J, Zhu J, Yin M, Xu M, Wang J, Huang N, Cui Z, Bian Z, et al: CircHMGCS1 promotes hepatoblastoma cell proliferation by regulating the IGF signaling pathway and glutaminolysis. Theranostics 9: 900-919, 2019.

41. Du WW, Yang W, Liu E, Yang Z, Dhaliwal P and Yang BB: Foxo3 circular RNA retards cell cycle progression via forming ternary complexes with p21 and CDK2. Nucleic Acids Res 44: 2846-2858, 2016

42. Tay Y, Rinn J and Pandolfi PP: The multilayered complexity of ceRNA crosstalk and competition. Nature 505: 344-352, 2014.

43. Zhang X, Luo P, Jing W, Zhou H, Liang C and Tu J: circSMAD2 inhibits the epithelial-mesenchymal transition by targeting miR-629 in hepatocellular carcinoma. OncoTargets Ther 11: 2853-2863, 2018.

44. Zhu Q, Lu G, Luo Z, Gui F, Wu J, Zhang D and Yong Ni Y: CircRNA circ_0067934 promotes tumor growth and metastasis in hepatocellular carcinoma through regulation of miR-1324/FZD5/Wnt/ $\beta$-catenin axis. Biochem Biophys Res Commun 497: 626-632, 2018.

This work is licensed under a Creative Commons Attribution-NonCommercial-NoDerivatives 4.0 International (CC BY-NC-ND 4.0) License. 